\title{
Design, Characterization, and Test of a Versatile Single- Mode Power-Over-Fiber and Communication System for Seafloor Observatories
}

Diouf Cherif 1, ", Quintard Veronique 1, Ghisa Laura 1, Guegan Mikael ${ }^{1}$, Perennou Andre 1, Gautier Laurent ${ }^{2}$, Tardivel Morgan ${ }^{2}$, Barbot Stephane ${ }^{2}$, Dutreuil Vincent ${ }^{2}$, Colas Florent ${ }^{2}$

${ }^{1}$ Ecole Nationale d'Ingénieurs de Brest, Lab-STICC CNRS UMR 6285, Technopôle Brest-Iroise, C.S. 73862, Brest Cedex 3 29238, France

2 IFREMER/RDT, Centre de Brest, BP 70, Plouzané 29280, France

*Corresponding author : Cherif Diouf, email address : c.diouf@yahoo.fr

\begin{abstract}
:
A power-over-fiber (PoF) and communication system for extending a cabled seafloor observatory is demonstrated in this contribution. The system allows the cabled seafloor observatory to be linked, through a single optical fiber, to a sensor node located $8 \mathrm{~km}$ away. The PoF system is based on an optical architecture in which power and data propagate simultaneously on the same single-mode fiber. The Raman scattering effect is exploited to amplify the optical data signals and leads to the minimization of the sensor node power consumption. Versatile low power electronic interfaces have been developed to ensure compatibility with a wide range of marine sensors. A low-consumption fieldprogrammable gate array and an energy-efficient microcontroller are used to develop the electronic interfaces. For an electrical input power of $31 \mathrm{~W}$, up to $190 \mathrm{~mW}$ is recovered at the sensor node while a data bitrate of up to $3.6 \mathrm{Mb} / \mathrm{s}$ is achieved. The PoF system has been tested and validated for turbidity and acoustic measurement applications. The current study focuses on the electronic development and the validation of the PoF system.
\end{abstract}

Keywords: Low power embedded systems, power-over-fiber (PoF), Raman amplification, remote sensing, seafloor observatories 
Cabled seafloor observatories are one of the key systems used to monitor the seabed environment. The observatories are able to supply low or high power marine instruments while providing quasi real-time, long-term monitoring of underwater environmental processes [1][2]. However due to their cabled nature, the observatories present a limited range of exploration and thus are less flexible than autonomous systems such as AUVs, UUVs or profiling floats. For further marine exploration close to the initial anchor point, some solutions based on copper cable extension, docking techniques [3] or mobile seafloor observatories [4] have been suggested in the literature. While these solutions can sometimes be cumbersome or costly to implement, the development we carried out is simple, lightweight, low cost and offers flexibility of deployment [5]. The solution is based on the Power-over-Fiber technology [6] which provides, through an optical fiber, power to a remote system.

Over the past few years, the technology regained interest due to progress achieved in High Power Laser Source (HPLS). The HPLS, are now able to deliver higher levels of output power while being designed in compact packages. This evolution has enabled new ranges of PoF applications. The PoF technology has the benefits of power transport without generating or without being affected by electromagnetic radiations. Thanks to its light weight, easy implementation and re-deployment, PoF can be an attractive power transport solution in unconventional or hostile conditions. It can constitute a good alternative to common power delivery methods relying on copper cables deployment. Hence the Power-over-Fiber technology has applications in domains such as high voltage power line monitoring [7, 8] thanks to its galvanic isolation. It can also be found in sensing systems for Internet of Things (IoT) [9] or even in high capacity Radio-over-Fiber transmission systems [10, 11, 12], where a composite optical/electrical cable can be replaced by an all in one optical fiber for powering up an RF antennas system.

In our context of seafloor exploration, the PoF system we have developed, permits to connect a cabled seafloor observatory box to a remote sensors hub through optical link (Fig. 1). A key feature of the system is that both data and power are carried out by one optical fiber, a standard fused silica single mode fiber (SMF-28). This is an important advantage in submarine domains where the number of optical interconnections should be limited as much as possible. Multiplexing power and data on the same fiber can be yet risky for the data transmission quality. Extensive analysis works were thus carried out in order to study power and data interactions on the fiber $[13,14]$. A first complete prototype $[15,16]$ was also set up to collect information from a hydrophone. The application was nevertheless very focused and limited. 


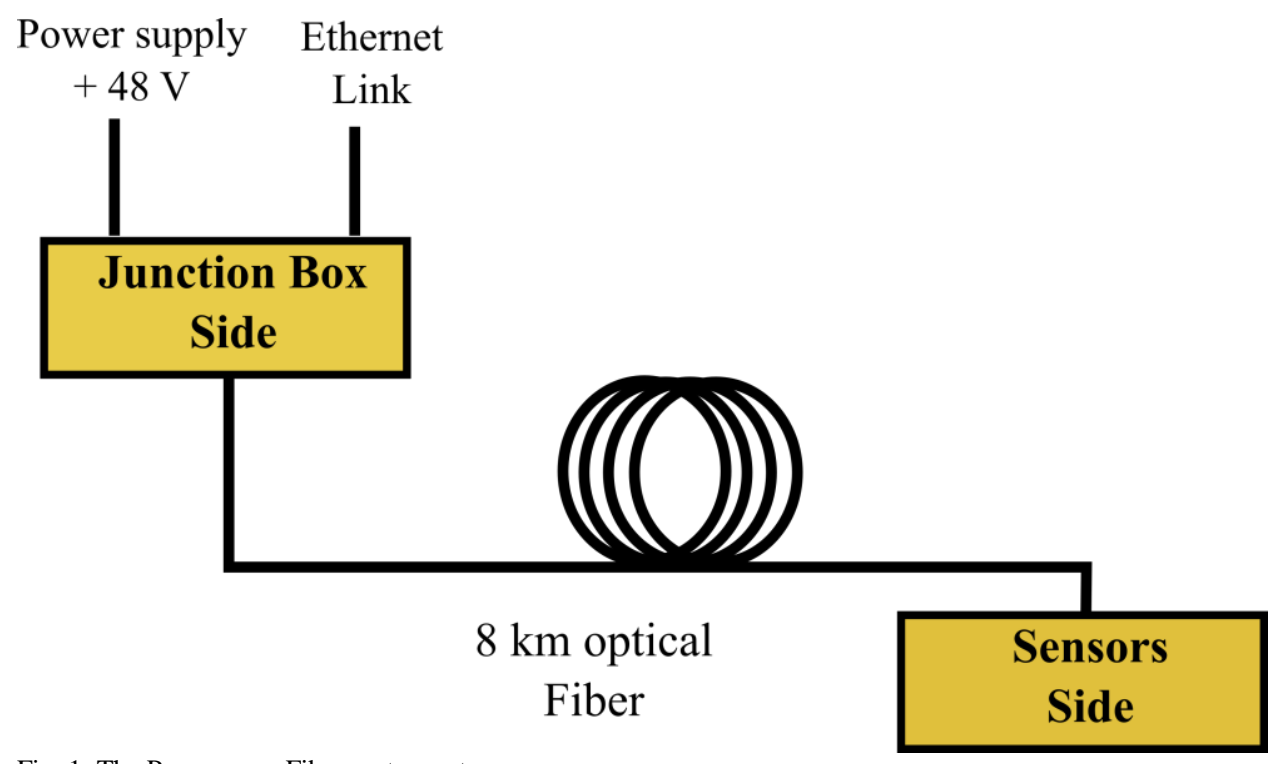

Fig. 1: The Power-over-Fiber system setup

We present, in this contribution, a versatile PoF system based on completely new electronic interfaces. The electronics presents significant improvements over the previous one, such as compatibility with various sensors, true bidirectional communication, quasi real-time operation and full user control. The design is based on a low power Field-Programmable-Gate-Array (FPGA) and an energy friendly microcontroller on each side of the optical fiber. Even in this advanced configuration, the system while being very versatile, has an acceptable power consumption. In addition, the new PoF prototype uses an $8 \mathrm{~km}$ marine optical cable specially designed for the Deep Sea Net observatory project [17].

The document is organized as it follows. First, the PoF optical architecture is introduced. Then the system is characterized to assess optical losses and optical power budget. Following these sections, the new electronic architecture will be presented, discussed and detailed. Test and system validation results in the case of low speed turbidity and high-speed acoustic applications are shown. Finally, conclusions are drawn and follow-up work is discussed.

\section{POF SYSTEM OPTICAL ARCHITECTURE PRESENTATION AND EXPERIMENTAL CHARACTERIZATION}

\section{A. System Architecture}

The optical architecture of the PoF system, presented in Fig. 2, is based on the work carried out in [13, 14]. Three optical wavelengths are multiplexed: $1537 \mathrm{~nm}, 1550 \mathrm{~nm}$ for the upstream and the downstream optical carriers, $1480 \mathrm{~nm}$ for the power transport. Along with power delivery, a bidirectional optical communication channel is thus implemented between the two sides of the system, the Junction box side and the sensors side (sensor node).

1) Junction Box Side 
The HPLS is integrated in the junction box side. This high-power source is a $1480 \mathrm{~nm}$ fibered Raman pump laser able to provide up to $10 \mathrm{~W}(40 \mathrm{dBm})$ of optical power. It should be noted that the $1480 \mathrm{~nm}$ wavelength was preferred over the more common $980 \mathrm{~nm}$ wavelength. This choice results from a tradeoff between transmission losses and far-end photovoltaic conversion efficiency. The $1480 \mathrm{~nm}$ wavelength source is actually allowing higher power recovery for a transmission distance greater than 1 $\mathrm{km}$. Compared to the architecture in [13, 14], an isolator has been added between the $1480 \mathrm{~nm}$ pump laser source and the multiplexer. The PoF system can now operate at higher levels of power ( $\geq 2.5 \mathrm{~W}, 34 \mathrm{dBm})$ while keeping a good quality of data
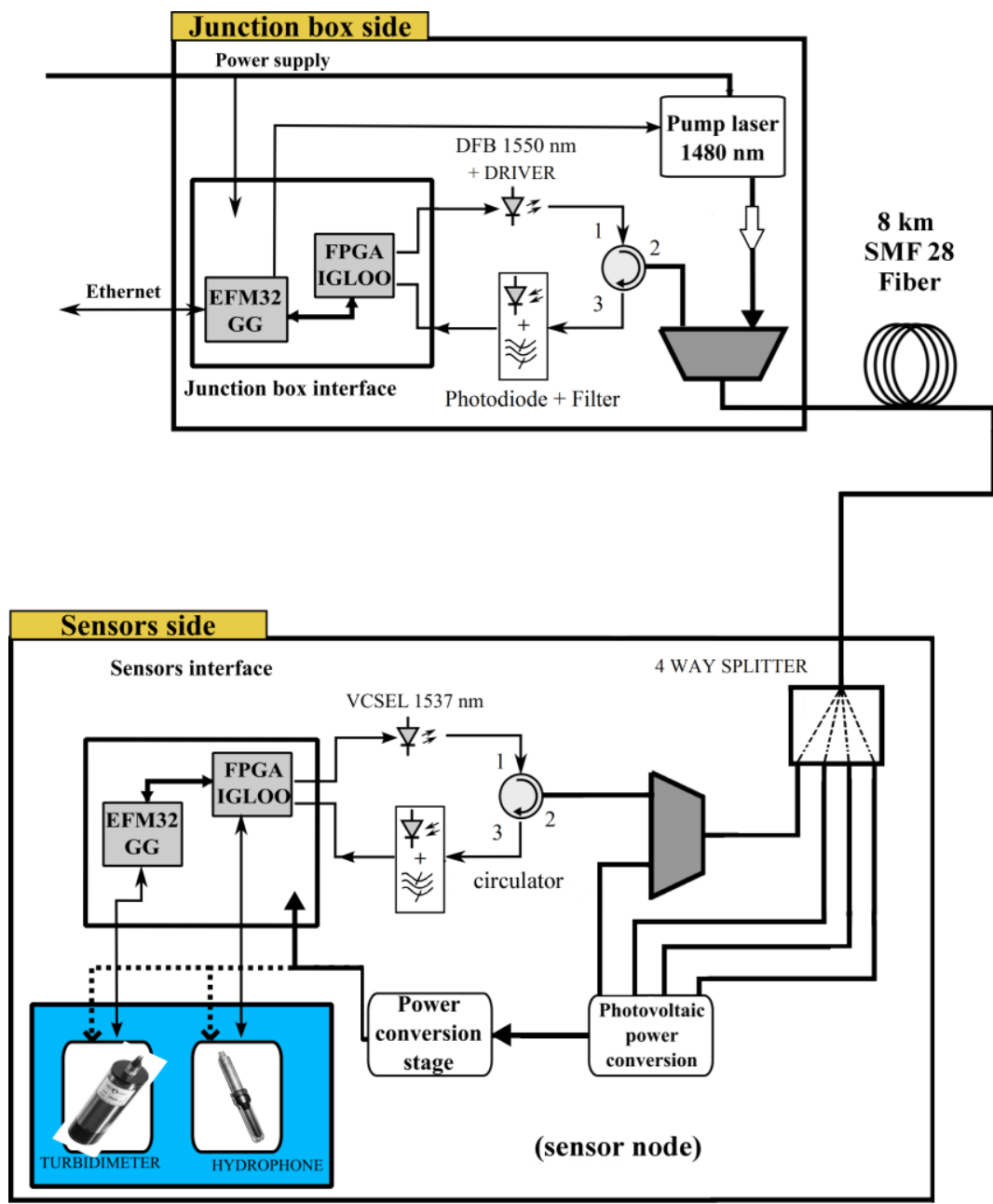

Fig. 2: System architecture illustration

used in order to reduce losses due to optical connectors but also for preventing damageable effects such as the Fiber Fuse problem [18].

Controlled by a MAX3668 laser driver IC, a 1550 nm DFB (Distributed Feedback Laser) is in charge of the downstream data signal transmission to the sensor node. The maximum output power of the laser is $10 \mathrm{~mW}$ (10 dBm). As shown in Fig. 2, an optical circulator is devoted to separate the $1550 \mathrm{~nm}$ downstream and the $1537 \mathrm{~nm}$ upstream data wavelengths. A circulator transmits an optical signal from one port to the next sequential port with a maximum intensity. Thus, the $1550 \mathrm{~nm}$ downstream signal entering 
port 1 exits port 2, while the $1537 \mathrm{~nm}$ upstream signal entering port 2 exits port 3 . Upstream data recovery is then carried out by a photodiode associated with a filter centered at $1537 \mathrm{~nm}$. Finally, bidirectional data and power channels are superimposed or separated by an optical multiplexer/demultiplexer ahead of the $8 \mathrm{~km}$ fiber

\section{2) Sensor node}

On the sensors side, a similar architecture is reproduced. The fiber is however fused to a 4 way splitter. This component distributes the received optical power on four 1400-1600 nm photovoltaic cells (PV) connected in parallel. This distribution scheme allows a higher conversion yield by avoiding cells saturation regime. The cells main role is to recover and supply electrical power to the node and to the sensors. A demultiplexer and a circulator guide the downstream data signal towards a photodiode associated with a $1550 \mathrm{~nm}$ centered filter. For upstream data transmission, a $1537 \mathrm{~nm}$ VCSEL (Vertical-Cavity Surface-Emitting Laser) is used. The VCSEL laser is operating without any specific laser driver.
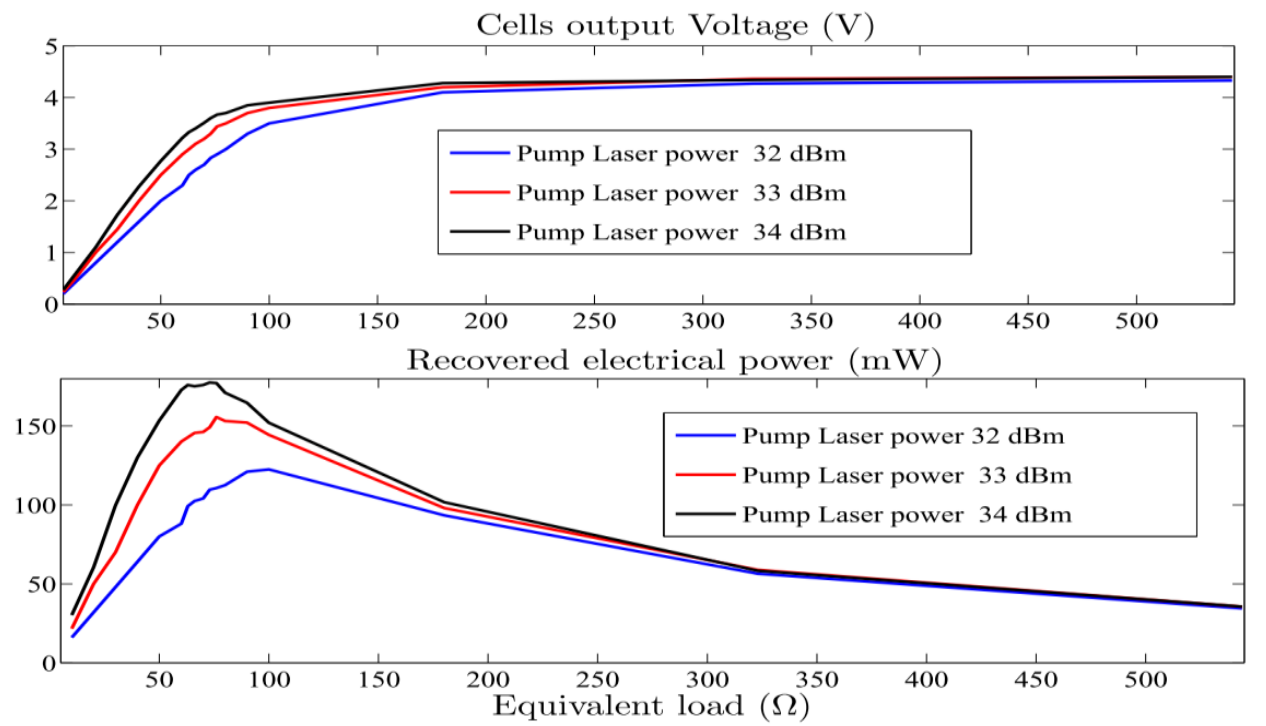

Fig. 3: Characterization of the $4 \mathrm{PV}$ cells associated in parallel a) Open circuit cells voltage, b) Recovered electrical power for different loading conditions.

\section{3) Optical linear and nonlinear effects on the $8 \mathrm{~km}$ fiber}

We have to consider several linear and nonlinear effects which can modify power and data transport efficiency along the optical fiber. An important effect is the optical fiber attenuation due to the $8 \mathrm{~km}$ length. Actually, a $0.5 \mathrm{~dB} / \mathrm{km}$ loss has been observed for our single mode fiber at the $1480 \mathrm{~nm}$ wavelength. It means that only less than $40 \%$ of the optical power generated by the pump laser is available at the sensor node. The second most significant effect is the Raman scattering [19]. This phenomenon generates an optical power transfer from the pump wavelength to a shifted wavelength. In our case with a pump laser centered at $1480 \mathrm{~nm}$, a part of the optical power is shifted around $1583 \mathrm{~nm}$ over near $40 \mathrm{~nm}$ (Amplified Spontaneous Emission - ASE). The main disadvantage of this phenomenon is a possible degradation of the bidirectional datalink quality due to the impact of the shifted optical power on the data wavelengths. The ASE can decrease upstream and downstream data OSNR (Optical Signal to Noise Ratio), leading to a degraded communication channel. 
Nevertheless Raman Effect has also its advantages, as a power transfer between the pump wavelength and data wavelengths is occurring. Hence, downstream and upstream optical data signals are amplified thanks to the stimulated Raman Scattering. Data losses from the fiber or from other optical components are partially compensated thanks to Raman amplification. This is a significant advantage for the low power sensor node. Meanwhile other optical effects such as Brillouin and Rayleigh scattering are occurring. They have however less influence than the fiber attenuation and the Raman scattering.

\section{B. Optical power characterization}

In order to assess power losses either for the 1480,1550 or $1537 \mathrm{~nm}$ wavelengths and to draw the final power balance, the system is statically characterized (i.e. all signals have a fixed continuous power). Along with the $8 \mathrm{~km}$ fiber losses, the following components induce different insertion losses depending on wavelengths. The insertion losses are between 0.3 and $1.3 \mathrm{~dB}$ for the Mux/Demux, 0.7 and $1.4 \mathrm{~dB}$ for the circulators, 0.8 and $1.2 \mathrm{~dB}$ for the optical filters. Furthermore the 4 way splitter generates losses of $0.3 \mathrm{~dB}$ at the wavelength of $1480 \mathrm{~nm}$ and around $6.3 \mathrm{~dB}$ for the data wavelengths. The few fusion splices are also sources of losses ( 0.1 to $0.3 \mathrm{~dB}$ per splice).

For the power link a total attenuation of $4.8 \mathrm{~dB}(67 \%)$ is measured from the pump to the PV cells. The $8 \mathrm{~km}$ optical fiber generates $4 \mathrm{~dB}$ of attenuation.

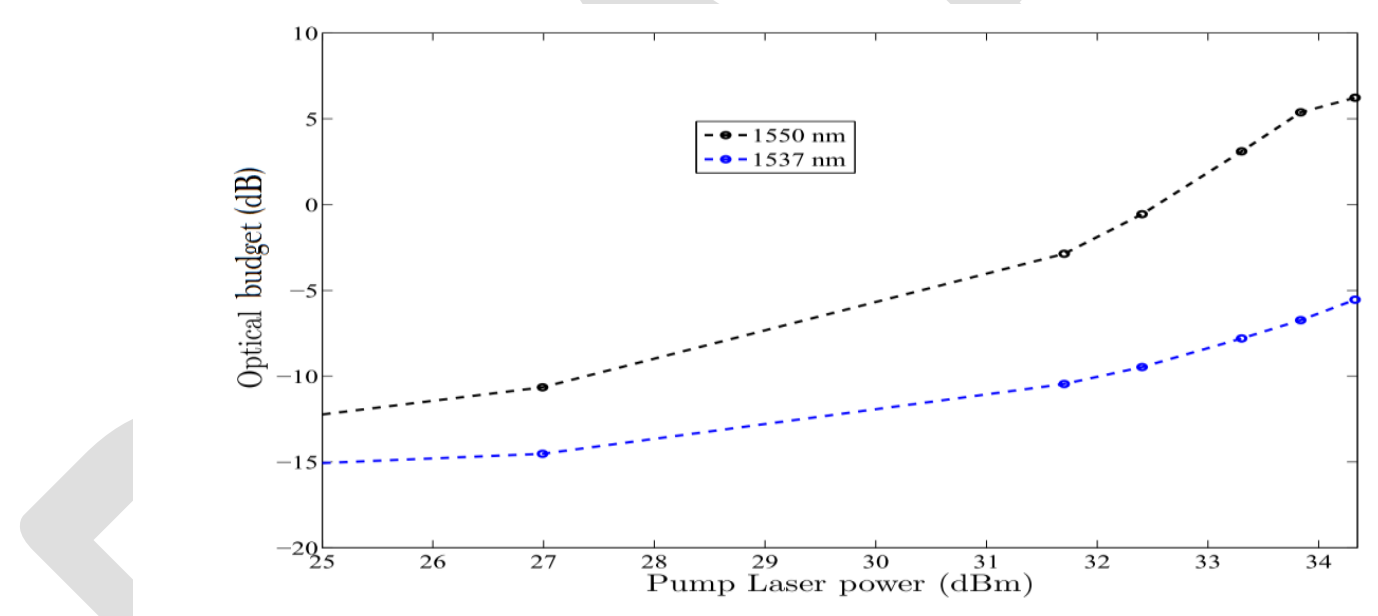

Fig. 4: Optical budget with respect to pump laser output power (HPLS).

The photovoltaic cells conversion efficiency depends on the loading impedance. Fig. 3 shows the electrical characterization of the 4 PV cells associated in parallel. These cells have an open circuit voltage of $4.2 \mathrm{~V}$ (Fig. 3a) and a maximum short circuit current of $15 \mathrm{~mA}$. They present an optimal conversion efficiency of $23 \%$. In these conditions, the electrical power available at the sensor node is about $7.6 \%$ of the HPLS output power. Electrical power measurements as a function of the load of the PV cells were made. Fig. $3 b$ shows the recovered electrical power according to equivalent resistive load. We can notice, that for a pump laser of 32,33 and $34 \mathrm{dBm}$, optimal power recovery is obtained when PV cells are respectively loaded by a 100,80 and $70 \Omega$ equivalent resistor. With these values, 120, 152 and $190 \mathrm{~mW}$ are available at the sensor node.

Raman pump lasers generally have low electrical to optical conversion efficiency (less than 10\%). Indeed, our HPLS has an efficiency of 6 to $7 \%$. For an output optical power of $33 \mathrm{dBm}$, the HPLS device requires at least $28.3 \mathrm{~W}$ (44.5 dBm) of electrical 
112

\section{5}

power. In Tab I, end-to-end electrical values are presented for a pump laser emitting an output optical power respectively of 32, 33 and $34 \mathrm{dBm}$.

TABLE I. END - TO-END ELECTRICAL POWER CONVERSION VALUES

\begin{tabular}{|c|c|c|}
\hline $\begin{array}{c}\text { HPLS electrical Input } \\
\text { Power }\end{array}$ & $\begin{array}{c}\text { HPLS optical Output } \\
\text { Power }\end{array}$ & $\begin{array}{c}\text { Recoverable electrical } \\
\text { Power at sensor node }\end{array}$ \\
\hline $25 \mathrm{~W}$ & $1.5 \mathrm{~W}$ & $120 \mathrm{~mW}$ \\
$(44 \mathrm{dBm})$ & $(32 \mathrm{dBm})$ & $(20.8 \mathrm{dBm})$ \\
\hline $28.3 \mathrm{~W}$ & $2 \mathrm{~W}$ & $152 \mathrm{~mW}$ \\
$(44.5 \mathrm{dBm})$ & $(33 \mathrm{dBm})$ & $(21.8 \mathrm{dBm})$ \\
\hline $31 \mathrm{~W}$ & $2.5 \mathrm{~W}$ & $190 \mathrm{~mW}$ \\
$(45 \mathrm{dBm})$ & $(34 \mathrm{dBm})$ & $(22.8 \mathrm{dBm})$ \\
\hline
\end{tabular}

Fig.4 shows the optical budget of the downstream and up-stream wavelenghts as a function of the pump power. Regarding the downstream datalink at $1550 \mathrm{~nm}$, without the presence of the HPLS power, $15 \mathrm{~dB}$ of optical losses are measured from the DFB output to the photodiode input (sensor node). In the presence of the HPLS power, the optical budget on the downstream datalink is clearly improving. For instance, with a $34 \mathrm{dBm}$ HPLS, the $15 \mathrm{~dB}$ losses are fully compensated and downstream data are even amplified by a $5.7 \mathrm{~dB}$ factor.

For the upstream link at $1537 \mathrm{~nm}$, higher optical losses are observed. A total attenuation of $16.7 \mathrm{~dB}$ is measured from the VCSEL output to the photodiode (Junction box side). The upstream link also benefits from the Raman amplification yet at lower level. In fact, in our setup, the Raman gain spectrum has its peak, around a wavelength of $1583 \mathrm{~nm}$. This value is closer to $1550 \mathrm{~nm}$ than to $1537 \mathrm{~nm}$, therefore a higher level of Raman gain is available for the downstream wavelength. Nevertheless, with a $34 \mathrm{dBm}$ HPLS power a gain of $10.3 \mathrm{~dB}$ is achieved for the $1537 \mathrm{~nm}$ upstream link. The upstream optical budget is then equal to $-6.4 \mathrm{~dB}$.

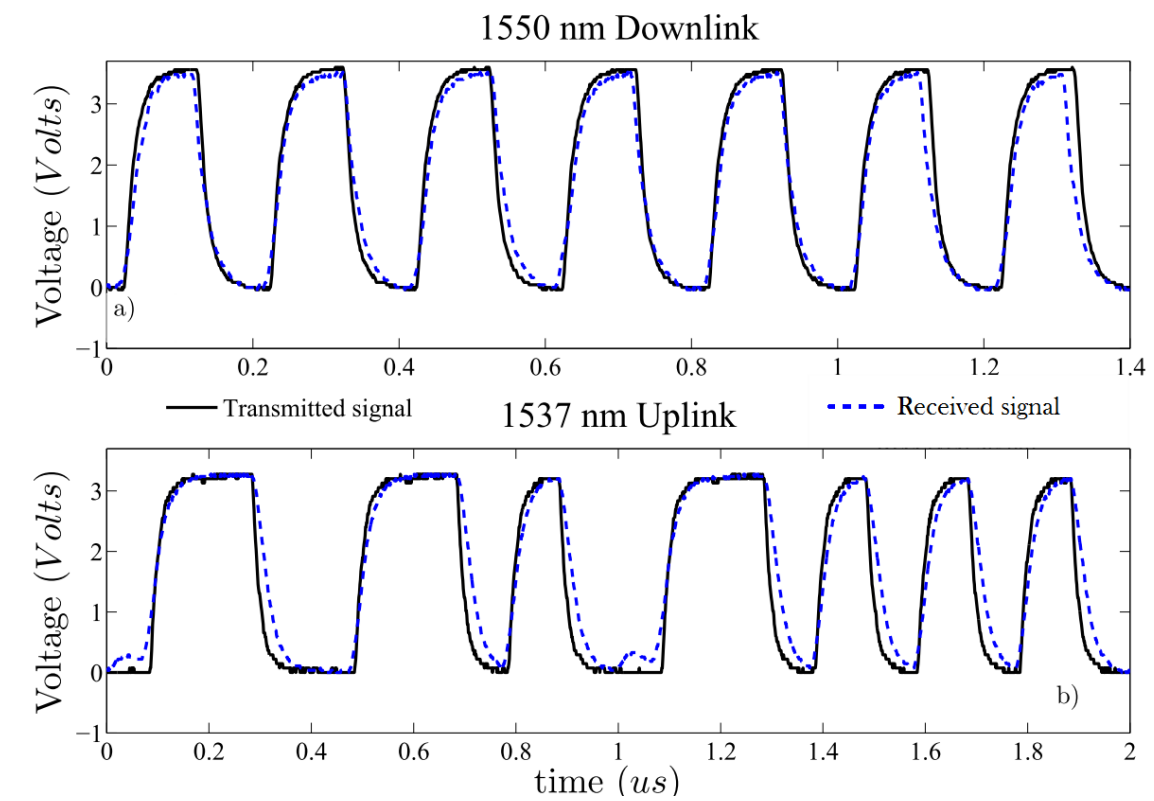

Fig. 5: a) Downstream signal sample (DFB optical power $1 \mathrm{~mW}, 0 \mathrm{dBm}$ ), b) Upstream signal sample (VCSEL optical power $0.5 \mathrm{~mW},-3 \mathrm{dBm})$ for a pump laser power of $33 \mathrm{dBm}(2 \mathrm{~W})$. 


\section{Datalink transmission quality assesment}

The data transmission quality can be estimated by using the Bit Error Rate (BER). In [13, 14], BER measurements have been presented for a similar optical architecture and a transmission distance of $10 \mathrm{~km}$. Results show that a minimum BER of 5.10 $0^{-7}$ was found for an input optical power of $33 \mathrm{dBm}$. The BER measurements were carried out at a bitrate of $150 \mathrm{Mbits} / \mathrm{s}$. With the current architecture (shorter fiber length and lower data bitrate), we can reasonably assume that we will get lower BER values. Here we briefly present in Fig. 5a, a downstream frame after O/E conversion at sensors side and in Fig. 5b an upstream frame after O/E conversion at the Junction box side, compared to the emitted signals respectively. The bitrate is $5 \mathrm{Mbits} / \mathrm{s}$. One should note that these waveforms will be also reconditioned by the FPGA input stages.

\section{ELECTRONIC DEVELOPMENT}

The characterization results obtained in the previous section are related to the $8 \mathrm{~km}$ fiber setup. For different operating conditions such as changes in input power, fiber type, fiber length or optical carriers, a new characterization may be required in order to draw reliable power and data figures. The current section will now deal with the PoF system electronic interfaces.

The electronic interface at the Junction box should allow a user to connect and interact with the PoF system, either for control, configuration or data acquisition. On the other hand, the electronic interface at the sensor node should be able to power and connect the marine sensors to PoF system. For proof-of-concept purposes, electronic interfaces at both sides were suggested and developed for a $5 \mathrm{~km}$ PoF application [15]. This PoF system was able to retrieve an acoustic signal from a passive hydrophone connected to the sensor node. As the application target was very specific, the system suffered from several limitations. Among them, we noticed the lack of true bidirectional communication. Once powered, the system would operate by itself and the data from the hydrophone were just up-streamed to the connected user. Neither additional data nor control frames could be sent to the sensor node. The system flexibility was thus very limited. Another major weakness was the lack of versatility in sensors type, only sensors similar to the hydrophone could be compatible with the system.

The new electronic design is based on the association of a low consumption FPGA and an energy-efficient microcontroller (MCU), both at the Junction box and the sensors interface. The architecture, presented in Fig.2, allows hardware (through the FPGA) and software (through the MCU) flexibility. This new architecture is particularly able to deal with low or high sampling rate sensors while meeting power constraints. 


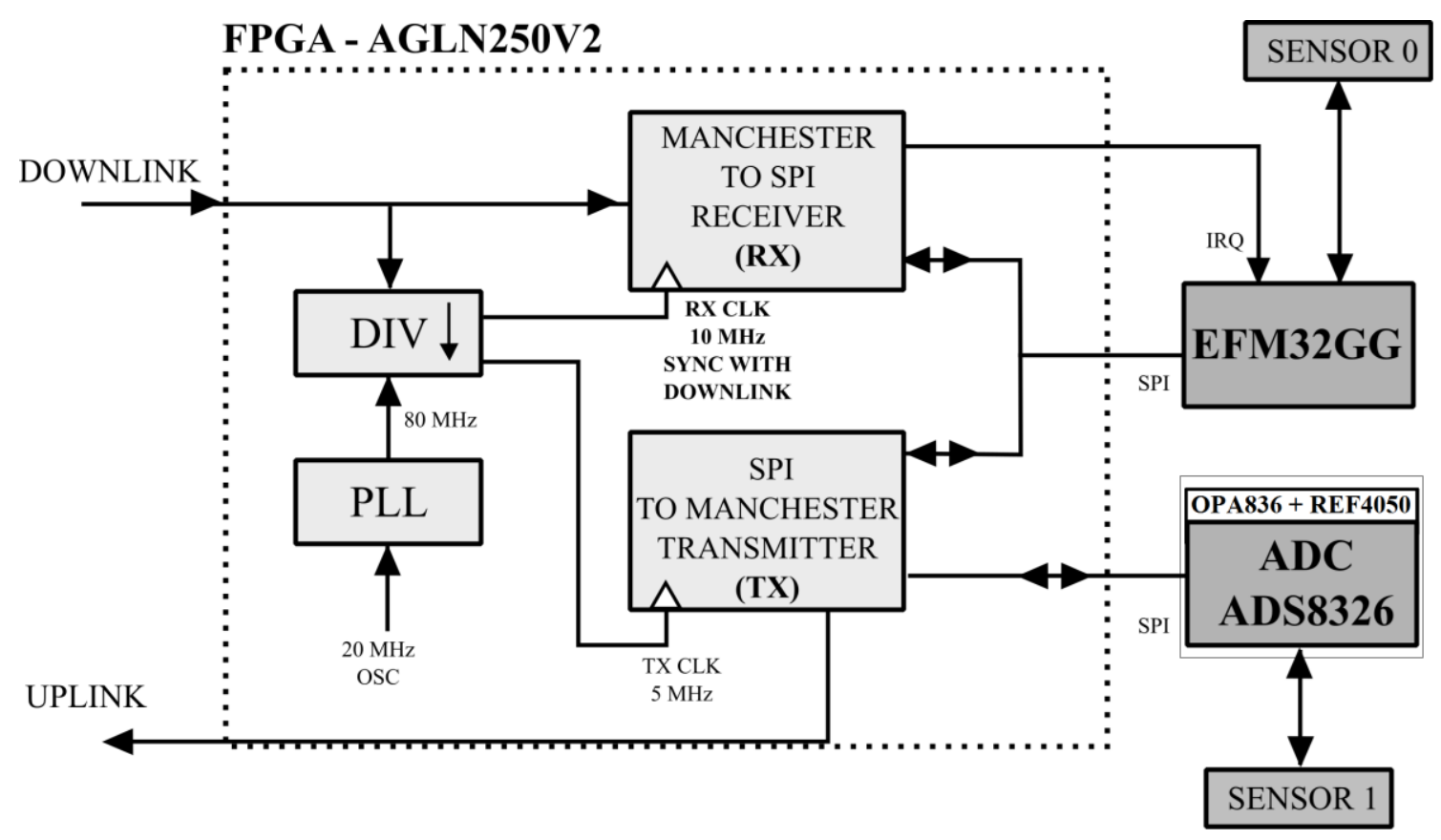

Fig. 6: FPGA - AGLN250V2 simplified architecture and its link with the EFM32 microcontroller (sensors side).

The EFM32 Giant Gecko (EFM32GG) is the microcontroller device used in the current PoF system. The EFM32GG is a 32-bit ARM Cortex M3 component developed by Silicon Labs. It presents a very low power consumption (around $1 \mathrm{~mW} / \mathrm{MHz}$ at $3.3 \mathrm{~V}$ ) and has a maximum operating frequency of $48 \mathrm{MHz}$, a $1 \mathrm{MB}$ of flash memory and $128 \mathrm{~KB}$ of RAM. The microcontroller has been already used in energy efficient life science monitoring [20] and is compatible with real time operating systems such as FreeRTOS [21]. The Microsemi's IGLOO nano Agln250v2 is the FPGA device used in the PoF system. The component has a native 36 kbits of RAM memory, a native PLL and a ROM memory of 1024 bit. The component is particularly suitable for very low power applications [22]. It has also been used as a programmable component in a $0.2 \mathrm{~km}$ power-over-fiber system [23].

Depending on the targeted application, a given sensor can be either connected to the FPGA or to the MCU. However, one of the microcontroller main advantages is that it is easy to interface. It can use standard communication protocols such as $\mathrm{I}^{2} \mathrm{C}$, MicroWire, SPI, Serial link (synchronous/asynchronous).

\section{A. Sensors interface}

The electronic architecture at the sensors interface is presented in Fig. 6. The EFM32GG and the FPGA communicate through a standard SPI link. The EFM32GG acts as master component for the sensor node. It waits for a downstream frame/command from the Junction box interface, to either apply a configuration such as a RESET command, to read a configuration or to activate/deactivate a given sensor. On the other hand, the FPGA Igloo mainly acts as a transmission/reception unit. Downstream Manchester data decoding and upstream Manchester data coding blocks are thus implemented in the FPGA. When a new downstream frame is available, an interrupt request (IRQ) is triggered by the FPGA in order to inform the microcontroller. The MCU can then pull the data and carry out the associated action.

One could also note, in Fig. 6, a direct SPI link between an external 16 bit Analog-to-Digital converter (ADC) block ADS8326 and the FPGA Manchester transmitter block. We have set up this configuration with the aim of interfacing the system 
with analog sensors requiring higher sampling rate such as hydrophones. Digital data from the analog-to-digital conversion block, are in this case directly transmitted by the FPGA. The microcontroller is thus bypassed, superfluous dead-times avoided and by so doing higher bitrates can be attained.

\section{B. Junction box interface}

A user should be able to control, through the Junction box interface, the whole PoF system. For this purpose, a telnet over TCP/IP server is implemented in the microcontroller. The EFM32GG does not have a native Ethernet peripheral. Hence, the ASIX AX88796C is used as an external Ethernet controller. Besides this TCP/IP link, an UDP/IP port is also available. This UPD/IP link will be used by the user to recover high bitrate data in a streaming mode of transfer.

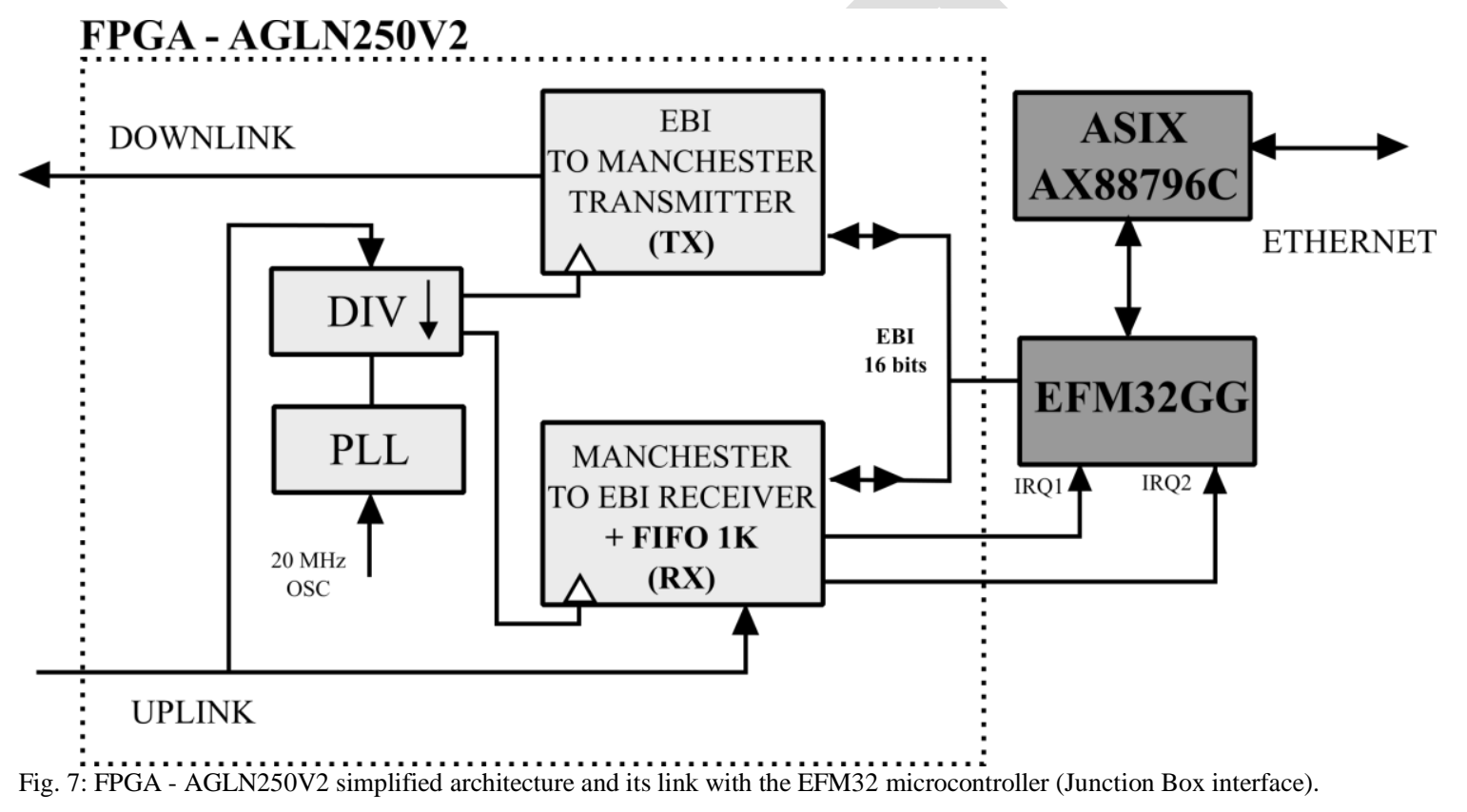

Fig. 7 is an illustration of the electronic architecture. This architecture is similar to the one presented in Fig 6. However, a 16bit parallel bus is now used between the FPGA and the EFM32GG. Downstream data transmission is triggered by the EFM32GG, which has beforehand written a 32-bit frame to the FPGA transmission block (two write sequences via the 16-bit bus). When the sensor node received this control frame, it answers with one 32-bit frame. Then at the junction box interface, an IRQ is triggered on the first channel IRQ1, allowing the EFM32GG to pull the 32-bit data (two read sequences) and to carry out the associated action.

The sensor measured data (upstream data) can be transmitted in 16-bit or 32-bit frame format depending on the type of sensors. For low data rate sensors 32-bit frames are used and for high data rate sensors 16-bit frames are used (streaming data transfer mode). In this latter case, at the junction box, the received data are successively saved to a 1024 bytes FIFO. When the FIFO is full, an IRQ, from the second channel IRQ2 (Fig. 7), is triggered allowing the EFM32GG to pull out the FIFO content and upstream the 1024 bytes length data to the user (UDP/IP streaming mode transfer). 


\section{Bidirectional datalink between the two interfaces}

The datalink between the two interfaces is a full duplex asynchronous link based on the Manchester coding scheme. A Manchester digital signal is generated by combining the data signal and the transmission clock signal. The numerous successive transitions on a Manchester signal allows a precise synchronization between the receiving clock and the incoming frames. Clock drift or high rate sampling, such as needed in serial links can be avoided. In the current application, the decoding/receiving clock frequency is twice the transmission frequency. From the FPGA $20 \mathrm{MHz}$ external oscillator, the native PLL is used to obtain an 80 MHz clock. The $80 \mathrm{MHz}$ output is then downscaled to generate the coding and the synchronized decoding clock (Fig. 6, 7). By default, the transmission/coding clock is set to $5 \mathrm{MHz}$ and the receiving/decoding clock is set to $10 \mathrm{MHz}$. For the latter, downscaling registers are associated with an edge detector [24]. This method allows the receiving clock to be always synchronized with incoming frames. Clock drift is avoided and precise data sampling is enabled.

Upstream or downstream data are transmitted with a " 1010 " binary header. The header allows the decoding clock at the opposite interface to be more easily synchronized. The exchanged data frames also embed a Cyclic-Redundancy-Check (CRC) for robust communication between the sensors interface and the Junction box interface. To distinguish two successive downstream or upstream frames a systematic dead-time of at least twice the transmission period is inserted between frames. With $5 \mathrm{MHz}$ as transmission frequency, the maximum data-rate, computed in streaming mode, is finally $3.6 \mathrm{Mbits} / \mathrm{s}$ for a 16 bits frame and 4.2 Mbits/s for a 32 bits frame. One could easily increase the bidirectional link bitrate by using higher transmission and receiving frequencies.

\section{Sensors interface power stage}

Sensors interface power conversion stage is composed of three specialized DC/DC converters and one supercapacitor for energy storage. The first DC/DC converter (Linear Technology LTC3426) provides a $+5 \mathrm{~V}$ voltage in order to supply the EFM32GG support board. This board also has a DC/DC step-down converter that is powering the EFM32GG core with a voltage of $+3.3 \mathrm{~V}$. A $+3.3 \mathrm{~V}$ voltage is also provided by the high efficiency TPS62203 from Linear Technology. This latter supplies the AGLN250V2 FPGA, electronic circuits interfacing the acoustic sensor with the FPGA and also the transimpedance amplifier (downlink O/E converter). A Texas Instruments LM27373 is finally available for providing a $+12 \mathrm{~V}$ supply for the turbidimeter and similar higher voltage sensors. Finally, the power stage is completed by a $2.5 \mathrm{~F}$ super-capacitor. The super-capacitor allows sensors requiring higher current inputs, to operate during short durations.

\section{E. Sensors interface power consumption}

The sensor node interface can be divided in 4 different power blocks: The EFM32GG block, the FPGA block, the optical components block and the sensors. The power consumption for the first three blocks is detailed in following paragraphs. The sensors block (turbidimetry and hydrophone sensors in our case) will be presented in the validation section.

\section{1) EFM32GG block}

The EFM32GG microcontroller has two active peripherals: the SPI peripheral in order to communicate with the FPGA. The EFM32GG also uses one of its serial RS232 peripheral in order to communicate, through a MAX3222 RS232 driver, with the turbidity sensor. It has also to be specified that the MCU is using an electronic support board causing extra power consumption. The MCU core is clocked thanks to a local RC circuit at $28 \mathrm{MHz}$. It consumes $1 \mathrm{~mW}$ per MHz. To limit power waste, the 
frequency will be downgraded to $7 \mathrm{MHz}$ ( $7 \mathrm{~mW}$ core consumption). Depending on the type or the rate of measurements, the clock frequency can be further decreased to $1 \mathrm{MHz}$ (1 mW consumption), or even to a lower frequency in order to optimize the CPU power consumption. This electronics block consumes $14 \mathrm{~mW}(11.4 \mathrm{dBm})$ for a $7 \mathrm{MHz}$ core frequency.

\section{2) FPGA block}

The FPGA is used from a modified starter kit from Microsemi. While the FPGA is a very low consumption component, the power hungriest element is the $20 \mathrm{MHz}$ external oscillator (starter kit). Under $+3.3 \mathrm{~V}$ supply, the oscillator requires $5 \mathrm{~mA}$, leading to a $16.5 \mathrm{~mW}$ consumption. The whole FPGA block consumes up to $32.5 \mathrm{~mW}(15.1 \mathrm{dBm})$.

\section{3) Optoelectronic components}

Besides the four photovoltaic cells that are providing the electrical power, other optical components are present: the VCSEL laser and a photodiode combined with a passive filter and a transimpedance amplifier (TIA: OPA836). The VCSEL is directly driven by a pin of the FPGA, no laser driver is used. For a high logical state the VCSEL output power is set to $-3 \mathrm{dBm}$. In this condition, for a $5 \mathrm{MHz}$ transmission frequency, the VCSEL power consumption is around $6 \mathrm{~mW}(7.8 \mathrm{dBm})$ and the TIA associated with the photodiode converter consume around $14 \mathrm{~mW}(11.4 \mathrm{dBm})$.

Tab. II summarizes the electrical power consumption of the sensor node for an EFM32GG CPU core clocked at $7 \mathrm{MHz}$ and a continuous transmission/reception at $5 \mathrm{MHz}$. In those conditions the power consumption is estimated to $66.5 \mathrm{~mW}$ (18.2 dBm). This estimation has been done with a frequency of the CPU compatible with many sensors. Obviously if the CPU frequency is increased, the power consumption will increase as well.

TABLE II. SENSOR NODE POWER CONSUMPTION FOR THE DIFFERENT BLOCKS

\begin{tabular}{|c|c|c|c|c|}
\hline $\begin{array}{c}\text { MCU block / CPU } \\
\text { @ } 7 \mathrm{MHz}\end{array}$ & FPGA block & $\begin{array}{c}\text { VCSEL@ } \\
5 \mathrm{MHz}\end{array}$ & $\begin{array}{c}\text { TIA and O/E converter } \\
\text { @ } 5 \mathrm{MHz}\end{array}$ & Total \\
\hline $\begin{array}{c}14 \mathrm{~mW} \\
(11.4 \mathrm{dBm})\end{array}$ & $\begin{array}{c}32.5 \mathrm{~mW} \\
(15.1 \mathrm{dBm})\end{array}$ & $\begin{array}{c}6 \mathrm{~mW} \\
(7.8 \mathrm{dBm})\end{array}$ & $\begin{array}{c}14 \mathrm{~mW} \\
(11.4 \mathrm{dBm})\end{array}$ & $\begin{array}{c}66.5 \mathrm{~mW} \\
(18.2 \mathrm{dBm})\end{array}$ \\
\hline
\end{tabular}

For test and experimental validation purposes, two sensors systems are integrated in the complete PoF prototype. The first sensor, a passive hydrophone, is connected to the FPGA, while the second one, a turbidimeter is connected to the EFM32GG (Fig. 2).

A. Acoustic measurements 


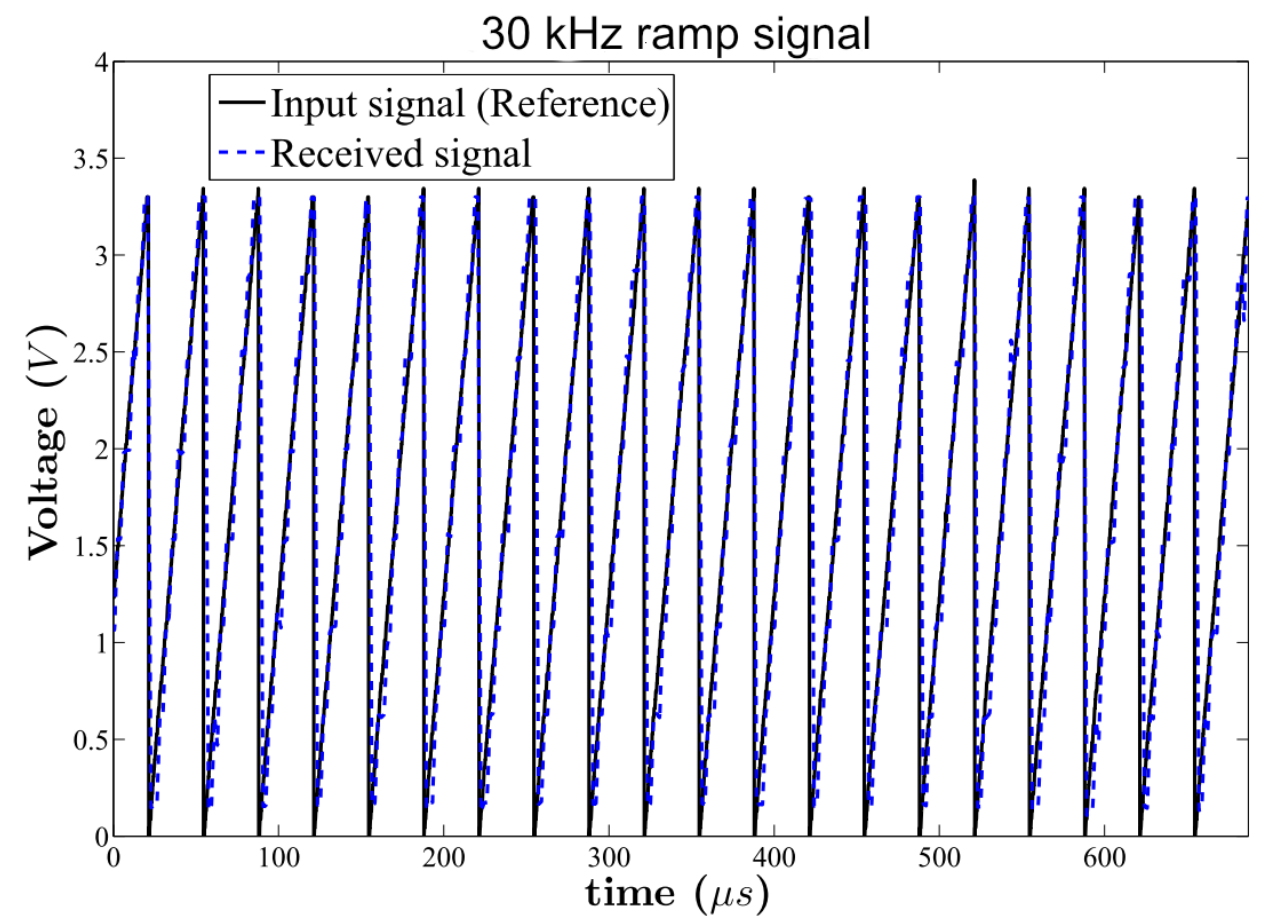

Fig. 8: Samples of a $30 \mathrm{kHz}$ ramp signal received at the Junction Box interface.

In this test configuration, a passive analog sensor presenting characteristics of a hydrophone or a geophone is connected to the 254 PoF system [25]. The bandwidth range is from a few $\mathrm{kHz}$ to $90 \mathrm{kHz}$. The upper frequency limit is set by the ADC maximum sampling frequency and the maximum bitrate on the bidirectional channel (3.6 Mbits/s). One possible configuration is to connect the device directly to the EFM32GG as the MCU has a native 12 bits analog-to-digital peripheral. However, the EFM32GG clock frequency has to be set as low as possible due to power waste consideration. Thus, a more efficient solution is to connect the sensor

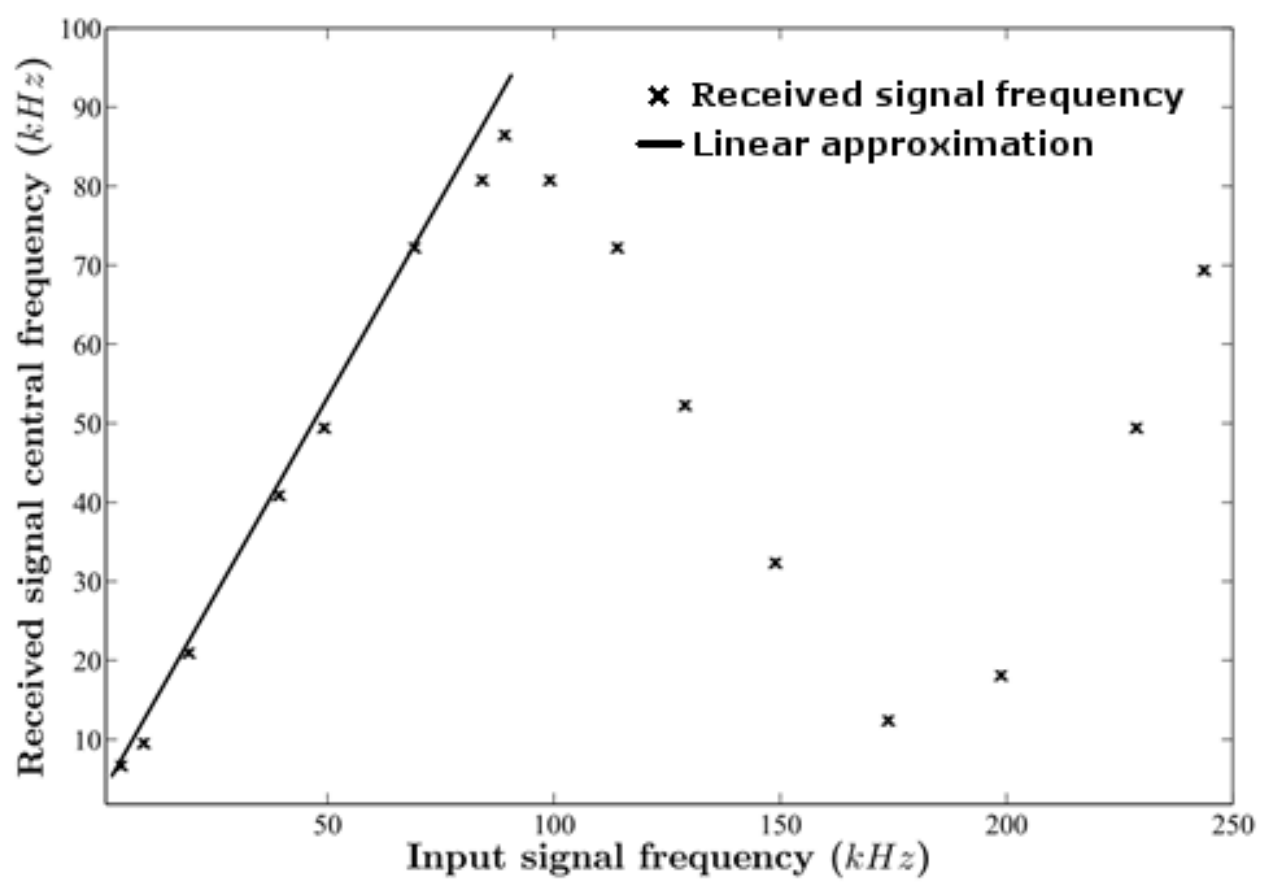

Fig. 9: System bandwidth characterization for acoustic application (anti-aliasing not applied). 
to the FPGA through an ADC converter. Sensor data can thus be directly upstreamed to the Junction box interface without MCU intervention.

The ADS8326 is the analog-to-digital converter in use. It is a low power 16 bits ADC, with $250 \mathrm{kSps}$. It is connected to the FPGA through a 3-wire SPI link. In such configuration the FPGA acts as a master component providing the SPI clock and the SPI Chip Select control signal and it recovers the converted analog data thanks to the SPI MISO signal (Fig. 7). The ADS8326 requires $22(6+16)$ clock cycles in order to provide a valuable 16-bit data. A high precision voltage reference (Texas Instruments REF5040) and an amplifier (Texas Instruments OPA386) are also present for robust analog acquisition. Tab. III summarizes the interfacing electronic power consumption. A total value of $23 \mathrm{~mW}(13.6 \mathrm{dBm})$ is obtained. Then a maximum of $89.5 \mathrm{~mW}(19.5 \mathrm{dBm})$ is obtained for the whole sensor node.

TABLE III. POWER CONSUMPTION OF THE HYDROPHONE ELECTRONIC MODULE

\begin{tabular}{|c|c|c|c|}
\hline AD8326 & OPA386 & REF5040 & TOTAL \\
\hline $10 \mathrm{~mW}$ & $11 \mathrm{~mW}$ & $2 \mathrm{~mW}$ & $23 \mathrm{~mW}$ \\
$(10 \mathrm{dBm})$ & $(10.4 \mathrm{dBm})$ & $(3.0 \mathrm{dBm})$ & $(13.6 \mathrm{dBm})$ \\
\hline
\end{tabular}

Data sampling is triggered by the user connected to the Junction box interface. To validate the PoF setup, the ADC converter is connected to a waveform generator. Ramp signals are generated and transmitted through the PoF system. An example of reference (sensors side) and received signals are plotted in Fig. 8 with a $30 \mathrm{kHz}$ frequency ramp signal. A good accordance can be found between the two signals.

The second validation involves the whole system bandwidth experimental characterization. A bandwidth of $90 \mathrm{kHz}$ is expected. Series of sinusoidal signals with frequency varying from $1 \mathrm{kHz}$ to $250 \mathrm{kHz}$ are generated and the digitalized signals are received by the user. In Fig. 9, the central frequency of the received signals is plotted with respect to the input signal frequency. Up to $90 \mathrm{kHz}$, the central frequency changes linearly with respect to the input signal frequency. As expected, from $90 \mathrm{kHz}$, the central frequency starts decreasing due to spectral overlapping.

\section{B. Turbidity measurements}

Turbidity represents the amount of cloudiness of a liquid such as water. The cloudiness is generally caused by foreign particulate matter suspended in the fluid. Turbidity measurements can provide valuable information regarding the liquid state and conditions. Turbidity measurements have application in several domains such as chemistry, biology, water treatment, sedimentation or pollution monitoring, etc... 
In the actual application case, we use a turbidity sensor, an "ECO NTU" from WET labs [26]. This sensor has a maximum sampling frequency of $8 \mathrm{~Hz}$. The turbidity values can be retrieved thanks to a RS232 serial link with the sensor which is connected to the EFM32GG through a MAX3222 driver.

When the turbidity sensor is activated, it requires $20 \mathrm{~mA}$ under $12 \mathrm{~V}$, thus $240 \mathrm{~mW}(23.8 \mathrm{dBm})$ over the measurement period. As this sensor needs more power than available at the node, $240 \mathrm{~mW}$ versus $190 \mathrm{~mW}$, a continuous measurement at $8 \mathrm{~Hz}$ is not possible. Periodic measurements can be however carried out over short durations. The whole sensor node will require around 300 $\mathrm{mW}(24.8 \mathrm{dBm})$ to correctly operate. The extra operating power needed will be provided by the $2.5 \mathrm{~F}$ supercapacitor added as an energy storage solution.

The turbidimeter should be turned off during 2 minutes between two measurements of 10 seconds. These 2 minutes allow the sensor node to recover its initial power operating point. At a higher rate, measurements consistency can be lost due to power fluctuations.

An experimental setup has been developed in order to validate the PoF system associated to the turbidity sensor. The setup main objective is to characterize the device located at $8 \mathrm{~km}$ far away from the Junction box interface. A single turbidity value is obtained after a 10 seconds measurement duration. The measurement is triggered by the user connected to the Junction box interface. The MCU then activates the sensor, receives and processes its measures before using the FPGA to upstream the data. The turbidity value which is coded on a 14-bit format is thus sent back from the sensor node to the user through the whole PoF system. The frame reply is on a 32-bit format, including the turbidity measure along with an error control check and the reply header.

The procedure of turbidimetry employed in this work consists in plunging and stabilizing the sensor in a clean water tank, then

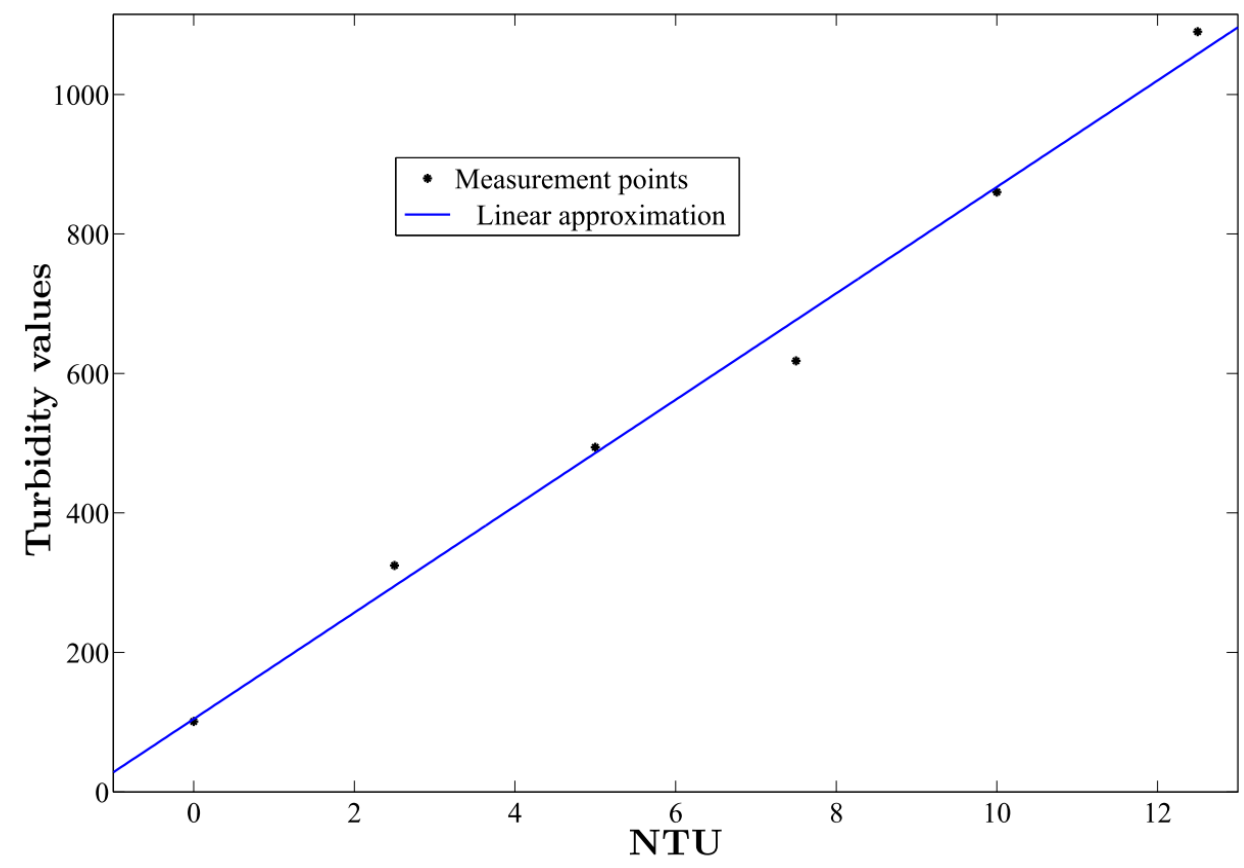

Fig. 10: Turbidity sensor characterization. present a linear increase with respect to the quantity of Formazine. For each step, a series of turbidity measurements is carried out and an average turbidity calculated. 
In Fig.10, we present turbidity evolution with respect to the volume of added Formazine. From the initial clean water (100 for turbidity value), five measurement points have been added. For each point a 2.5 Nephelometric Turbidity Unit (NTU) is added up until 12.5 NTU is reached. As expected, a quasi-linear evolution of turbidity, can be observed. The evolution can be compared to the linear approximation of the turbidity values.

The current PoF system can be a good solution to carry out turbidimetry or more generally optode based sensing at a remote area kms away from the seabed observatory anchor point. Although the "ECO NTU" requires more power than recoverable at sensors side, measurements can be correctly carried out by using local energy storage solution.

\section{CONCLUSION}

A Power-over-fiber system has been presented in this contribution. The galvanically isolated system is able to power a remote sensor hub, located a few kilometers away, while maintaining a bidirectional communication channel between its two sides. Another advantage of the system relies on concurrent transport of both data and power on the same optical fiber. This configuration allows to limit the number of required interconnections, making the system suitable for easy deployment in submarine domain. The prototype is able to deliver up to $190 \mathrm{~mW}(22.8 \mathrm{dBm})$ to the sensors side for a HPLS source of $2.5 \mathrm{~W}$ ( 34 $\mathrm{dBm})$ and accordingly a $31 \mathrm{~W}(45 \mathrm{dBm})$ of electrical input power. The electronic interfaces on both sides of the fiber are based on the association of a low power FPGA and an energy-friendly microcontroller. The association allows the PoF system to be flexible both in hardware and software. The system has been tested and validated with two different sensors. The first system plays the role of an analog acoustic sensor continuously emitting data. Measured data are transmitted in a streaming mode. Good accordance between generated and received waveforms is obtained. The whole PoF prototype has a $90 \mathrm{kHz}$ bandwidth. The second sensor is a turbidity sensor, which is operating at very low frequency compared to the first one. The turbidimeter requires more continuous power than the PoF system can provide. Energy storage is thus required, at the sensor node, in order to carry out turbidimetry. As a follow-up work, the system will be particularly tested and deployed for seabed monitoring in real life conditions.

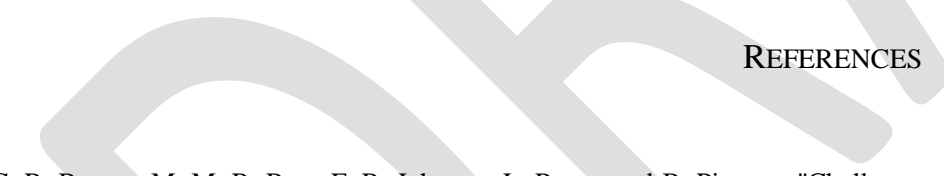

[1] C. R. Barnes, M. M. R. Best, F. R. Johnson, L. Pautet and B. Pirenne, "Challenges, Benefits, and Opportunities in Installing and Operating Cabled Ocean Observatories: Perspectives From NEPTUNE Canada," in IEEE Journal of Oceanic Engineering, vol. 38, no. 1, pp. 144-157, Jan. 2013.

[2] R. A. Petitt et al., "The Hawaii-2 Observatory," in IEEE Journal of Oceanic Engineering, vol. 27, no. 2, pp. 245-253, Apr 2002.

[3] B. W. Hobson et al., "The Development and Ocean Testing of an AUV Docking Station for a 21" AUV," OCEANS 2007, Vancouver, BC, 2007, pp. 1-6.

[4] K. Kawaguchi, K. Hirata, T. Nishida, S. Obana and H. Mikada, "A new approach for mobile and expandable real-time deep seafloor observation - adaptable observation system," in IEEE Journal of Oceanic Engineering, vol. 27, no. 2, pp. 182-192, Apr 2002.

[5] J. K. Choi, T. Yokobiki and K. Kawaguchi, "ROV-Based Automated Cable-Laying System: Application to DONET2 Installation," in IEEE Journal of Oceanic Engineering, vol. PP, no. 99, pp. 1-12.

[6] J.-G. Werthen, S. Widjaja, T.-C. Wu, J. Liu, "Power over fiber: a review of replacing copper by fiber in critical applications," Proc. SPIE 5871, Optical Technologies for Arming, Safing, Fuzing, and Firing, 58710C, doi:10.1117/12.619753, August 18, 2005.

[7] R. P. de Oliveira, F. V. B. de Nazaré and M. M. Werneck, "Development of a fiber Bragg grating single-point temperature sensor based on fixed filter demodulation technique," 2013 IEEE International Instrumentation and Measurement Technology Conference (I2MTC), Minneapolis, MN, 2013, pp. 10121016.

[8] X. Zhang et al., "A Gate Drive With Power Over Fiber-Based Isolated Power Supply and Comprehensive Protection Functions for 15-kV SiC MOSFET," in IEEE Journal of Emerging and Selected Topics in Power Electronics, vol. 4, no. 3, pp. 946-955, Sept. 2016. 
[9] J. Wang et al., "Power-over-fiber technique based sensing system for internet of things" 2016 15th International Conference on Optical Communications and Networks (ICOCN), Hangzhou, pp. 1-3, 2016.

[10] T. Umezawa et al., "100-GHz Fiber-Fed Optical-to-Radio Converter for Radio- and Power-Over-Fiber Transmission," in IEEE Journal of Selected Topics in Quantum Electronics, vol. 23, no. 3, pp. 23-30, May-June 2017.

[11] M. Matsuura and J. Sato, "Bidirectional Radio-Over-Fiber Systems Using Double-Clad Fibers for Optically Powered Remote Antenna Units," in IEEE Photonics Journal, vol. 7, no. 1, pp. 1-9, Feb. 2015.

[12] Y. Lee, K. Suto, H. Nishiyama, N. Kato, H. Ujikawa and K. I. Suzuki, "A novel network design and operation for reducing transmission power in cloud radio access network with power over fiber," 2015 IEEE/CIC International Conference on Communications in China (ICCC), Shenzhen, pp. 1-5, 2015.

[13] F. Audo, S. Perhirin, V. Quintard, M. Guegan, A. Perennou, and Y. Auffret, "Raman Amplification in Optically High-Powered Data Link Dedicated to a 10km Long Extension for Submarine Cabled Observatories," in Journal of Optics on IOPscience, Apr. 2013.

[14] Frédéric Audo, "Power and data over fiber for seafloor observatories" PhD dissertation. tel-00782502v3 Université de Bretagne Occidentale - Brest, 2012.

[15] S. Perhirin et al., "A power-over-fiber system and its low consumption remote equipment for submarine applications," 2013 MTS/IEEE OCEANS - Bergen, Bergen, pp. 1-6, 2013.

[16] F. Colas, S. Perhirin, F. Audo, S. de Blasi, C. Diouf, M. Guegan, L. Ghisa, V. Quintard, A. Perennou, J.F. Rolin, "Power and data over fiber for sea-floor observatories" Suboptic 2016, Dubai, UAE, April 2016.

[17] P. Valdy, V. Ciausu, P. Leon, P. Moriconi, V. Rigaud, et al., « Deep Sea Net: an affordable, and expandable solution for deep sea sensor networks », in IEEE Symposium on Underwater Technology and Workshop on Scientific Use of Submarine Cables and Related Technologies, Tokyo, Japon, 2007, pp. $172-175$.

[18] J.-G. Werthen, S. Widjaja , T.-C. Wu, J. Liu, "Power over fiber: a review of replacing copper by fiber in critical applications". Proc. SPIE 5871, Optical Technologies for Arming, Safing, Fuzing, and Firing, 58710C, doi:10.1117/12.619753, August 18, 2005.

[19] Headley C, Agrawal G P 2005 Raman Amplification in Fiber Optical Communications Systems (San Diego: Elsevier Academic Press)

[20] P. Passow, N. Stoll,S. Junginger and K. Thurow, "A wireless sensor node for long-term monitoring in life science applications," 2013 IEEE International Instrumentation and Measurement Technology Conference (I2MTC), Minneapolis, MN, 2013, pp. 898-901.

[21] C. S. Stangaciu, M. V. Micea and V. I. Cretu, "Hard real-time execution environment extension for FreeRTOS," 2014 IEEE International Symposium on Robotic and Sensors Environments (ROSE) Proceedings, Timisoara pp. 124-129, 2014.

[22] M. Dreschmann et al., "Reconfigurable Hardware for Power-over-Fiber Applications," 2010 International Conference on Field Programmable Logic and Applications, Milano, pp. 525-531. doi: 10.1109/FPL.2010.104., 2010.

[23] F.K. Lau, B. Stewart and D. McStay,“An optical powered subsea video monitoring system.” In Proc. SPIE 8372,Ocean Sensing and Monitoring IV, p.837209-837209, June 2012.

[24] M. Banu and A. E. Dunlop, "Clock recovery circuits with instantaneous locking," in Electronics Letters, vol. 28, no. 23, pp. $2127-2130,5$ Nov. 1992.

[25] Puillat Ingrid et al. Open-sea observatories: a new technology to bring the pulse of the sea to human awareness . In Oceanography, Prof. Marco Marcelli (Ed.), ISBN: 978-953-51-0301-1, 2012.

[26] Barnes, C.R., Best, M.M.R ., Zielinski, A., 2008. The NEPTUNE Canada regional cabled ocean observatory. Sea Technology 49, 10-14. 